\title{
Cooperation, social networks, and the emergence of leadership in a prisoner's dilemma with adaptive local interactions
}

\author{
Martín G. Zimmermann ${ }^{1,2,3, *}$ and Víctor M. Eguíluz ${ }^{1, \dagger}$ \\ ${ }^{1}$ Instituto Mediterráneo de Estudios Avanzados IMEDEA (CSIC-UIB), E-07071 Palma de Mallorca, Spain \\ ${ }^{2}$ Departamento de Física, Universidad de Buenos Aires, Buenos Aires, Argentina \\ ${ }^{3}$ Santa Fe Institute, 1399 Hyde Park Road, Santa Fe, New Mexico 87501, USA
}

(Received 23 September 2004; revised manuscript received 30 August 2005; published 16 November 2005)

\begin{abstract}
Cooperative behavior among a group of agents is studied assuming adaptive interactions. Each agent plays a Prisoner's Dilemma game with its local neighbors, collects an aggregate payoff, and imitates the strategy of its best neighbor. Agents may punish or reward their neighbors by removing or sustaining the interactions, according to their satisfaction level and strategy played. An agent may dismiss an interaction, and the corresponding neighbor is replaced by another randomly chosen agent, introducing diversity and evolution to the network structure. We perform an extensive numerical and analytical study, extending results in M. G. Zimmermann, V. M. Eguíluz, and M. San Miguel, Phys. Rev. E 69, 065102(R) (2004). We show that the system typically reaches either a full-defective state or a highly cooperative steady state. The latter equilibrium solution is composed mostly by cooperative agents, with a minor population of defectors that exploit the cooperators. It is shown how the network adaptation dynamics favors the emergence of cooperators with the highest payoff. These "leaders" are shown to sustain the global cooperative steady state. Also we find that the average payoff of defectors is larger than the average payoff of cooperators. Whenever "leaders" are perturbed (e.g., by addition of noise), an unstable situation arises and global cascades with oscillations between the nearly full defection network and the fully cooperative outcome are observed.
\end{abstract}

DOI: 10.1103/PhysRevE.72.056118

PACS number(s): 89.75.Hc, 02.50.Le, 87.23.Ge, 89.65.-s

\section{INTRODUCTION}

The emergence of cooperation in a group of agents has been traditionally discussed in the past using the Prisoner's Dilemma (PD) game [2]. In Ref. [3] it is shown how cooperation may be sustained by reciprocity in a population of agents meeting repeatedly with a certain degree of rationality. In evolutionary game theory, one general assumption is that players do not have any preference with whom to play, thus they are randomly selected and matched. However, in many social and economic environments this assumption does not hold and agents in fact display repeated encounters with only a subset of a large population [4]. This specific feature is naturally modeled with the aid of a network, where economic agents sit and interact only with their neighbors. This work will focus on the dynamics of the PD played on a network, with the attractive feature that the network itself is allowed to adapt according to the strategy and satisfaction level of the individual agents. Examples of other work using a network of interaction as a fundamental constituent range from herd behavior and information dispersal in financial markets [5-7], market-structure modeling [8], and firm competition in an oligopoly $[9,10]$.

Studies using PD games with fixed local interactions have shown that cooperation could be established for a range of parameters, before defection invades the whole population [11-17]. However, it is well known that in many social systems individuals are continuously creating or suppressing in-

\footnotetext{
*Electronic address: zeta@df.uba.ar

†Electronic address: victor@imedea.uib.es
}

teractions according to the benefits of the relationship. Although in some situations this phenomenon may occur in a slower time scale than the strategic dynamics and on a first approximation one is tempted to ignore such "degree of freedom," when repeated encounters turn out to be long term, then the network adaptation process must be included in the model and analyzed. One example where this problem might have relevance occurs in scientific collaboration networks [18], where scientists usually work in small groups of collaborations, and the relationships evolve under performance and self-interest of the individual members [19,20].

This paper addresses the problem of how cooperation may be sustained when local interactions evolve. Our results indicate that cooperation may be sustained as a result of heterogenous neighborhoods which arise in the dynamical evolution [1,21]. In particular, a PD game on an adaptive network is studied, where cooperation can be promoted and sustained by local interactions and a network adaptation process. Our contribution follows the bounded rationality paradigm. This includes a limited capacity to anticipate future opponents strategies, and in particular the network adaptation deals with the following assumptions: (i) depending on the strategy played and a satisfaction criteria, every link is evaluated for continuing or suppressing the interaction, (ii) individuals are not capable of computing a priori the best response as to whom connect, and they instead rely on a random selection of new partners, (iii) in the expectation of obtaining a benefit, individuals a priori always accept the proposed new interactions with new neighbors, (iv) the total number of links in the network is conserved. The latter point assumes a constrained resource environment, and introduces a limitation into the possible network configurations. 
The proposed game considers that each agent either plays cooperation $(C)$ or defection $(D)$ with all its local neighbors, as prescribed by the interaction network. In other words, we do not take into account memory and we label each agent as being either a $C$ or $D$ agent. The strategy is revisited each time step by imitating the neighbor's strategy with highest aggregate payoff. This very simple strategy update algorithm has been already shown to promote cooperation in some region of the PD parameter space [12-17]. The network dynamics considered here will only affect links between $D$ agents. We argue that these links are "disliked" by both agents, so given a certain probability, one of the agents may exchange the neighbor with a randomly chosen agent from the whole network. In other words, the network adaptation drives unsatisfied $D$ agents to "search" for other $C$ agents to exploit.

We have established results on the existence and stability of steady states. By steady state we refer to a state of the system where the network and strategies remain stationary. These states are composed of chains of cooperators, with defectors only linked to $C$ agents. In order to understand the robustness of the steady states, and the evolution of the network, we have also studied how perturbations affect these equilibria. We show how perturbations may actually grow, maintain, or destroy the chains of cooperators. These perturbations can lead to global oscillations between states with a large fraction of cooperators and another state with a large fraction of defectors, or even a complete all-defector state.

The emergence of global cooperative steady states is found to depend exclusively on the survival of certain $C$ agents with maximum payoff in the network, which we label $C$ leaders. More precisely, role differentiation has been shown to be an important outcome of the network adaptation [22]. We establish conditions for the evolution of the payoff of $C$ leaders and wealthy $C$ agents which is directly connected to the same network adaptation performed by $D$ agents. A rich dynamics arises in a certain region of parameters, where a competition between $C$ and $D$ leaders is observed. A close study of this competition shows that there are certain perturbations which induce global cascades in the system, where $C$ leaders may be destroyed. The asymptotic outcome of whole population depends crucially on the survival of $C$ leaders, and a full-defective network is reached when all $C$ leaders disappear.

With the aid of extensive numerical simulations we study how cooperation performs for a wide range of networks and PD parameters. Previous results with fixed networks [12-17], have shown that cooperation dominates up to a critical value of the PD parameters, where defection invaded the whole system. In our case, depending on the initial conditions, either the cooperative steady state or the all- $D$ network is reached. We find that in general the average payoff of $D$ agents is larger than that of $C$ agents, which also points in the direction that cooperation is sustained mainly due to the $C$ leaders. Finally, we study the robustness of steady states by introducing exogenous perturbations, such as errors in the imitation of a strategy. We find that these exogenous perturbations affect the survival probability of $C$ leaders. The network is robust up to a certain noise threshold, after which a full-defective outcome is reached.
The paper is organized as follows. In Sec. II we define the evolutionary network model. Section III describes the formal analysis, including steady state solutions and stability, as well as a result on the payoff evolution of $C$ leaders. In Sec. IV we show numerical experiments describing how a highly cooperative state is reached, topological properties of the asymptotic networks, a detailed explanation for the large cascades observed for certain parameters, and a robustness study when exogenous perturbations are allowed. Finally in Sec. V we discuss our results and open problems.

\section{THE MODEL}

We consider a game where $N$ agents play the Prisoner's Dilemma on a network $\mathcal{N}$. Each agent $i$ plays with a subset known as the neighbors, which will be allowed to evolve as specified below. Each interaction among two agents is represented in $\mathcal{N}$ by an undirected link, and the neighbors of agent $i$ will be denoted by $\mathcal{V}(i) \in \mathcal{N}$. An important parameter of the network is the average degree $K$, which corresponds to the average number of incidents links to an agent. We consider in general networks where the total number of agents $N$ is much larger than the typical degree $K$.

Let us denote by $s_{i}$ the strategy of agent $i$, where $s_{i}=1$ corresponds to play cooperation $(C)$, and $s_{i}=0$ corresponds to play defection $(D)$. These will be referred generically as $C$ agents or $D$ agents, respectively. The payoff matrix for a two-agent PD interaction is

$$
\begin{array}{c|c|c} 
& C & D \\
\hline C & \sigma, \sigma & 0, b \\
\hline D & b, 0 & \delta, \delta
\end{array}
$$

where $b>\sigma>\delta>0$, and $b / 2<\sigma$. For the remainder of the paper we take $\sigma=1, \delta=0.1$, and the incentive to defect, $b$, is restricted to $1<b<2$.

As discussed in the Introduction, we follow the bounded rationality paradigm. Agents are assumed not to have the computational power to anticipate strategic moves of opponents (agents have no memory), nor compute a priori the best agent to whom connect. Their rationality only allows them to play the same strategy with all its neighbors, observe the aggregate payoff of its neighbors and its strategy (from the payoff obtained), and whenever the agents changes opponents they rely on a random selection of new partners. In this context we let the agents update both their strategy and their local neighborhoods $\mathcal{V}(i)$ by a very simple prescription. The game occurs in discrete time $t$ and each step is divided into three stages. Step (i): Every agent plays the PD game with its neighbors. Step (ii): Every agent imitates the strategy of the best neighbor. Step (iii): Every agent applies a network dynamics which adapts its local neighborhood.

We use a synchronous update, where all the agents decide at the same time their strategy and their respective new neighborhood for the next time step. Alternatively, asynchronous update [23] corresponds to select in each time step a single random agent, and perform the above steps with data corresponding at that time of update.

The first two stages are straightforward and follow Ref. [15]: 
Step (i): Each agent $i$ plays a PD game with each neighbor using the same strategy $s_{i}$, and collects an individual payoff $\Pi_{i}$ for time $t$,

$$
\Pi_{i}(t)=s_{i} \mu_{i} \sigma+\left(1-s_{i}\right)\left[\mu_{i} b+\left(K_{i}-\mu_{i}\right) \delta\right]
$$

where $K_{i}$ is the degree of agent $i$ and $\mu_{i}$ is the number of $C$ neighbors of agent $i$ :

$$
\mu_{i}=\sum_{j \in \mathcal{V}(i)} s_{j} .
$$

Step (ii): Each agent $i$ revisits its current strategy by imitating the neighbor's strategy with the highest aggregate payoff. We introduce the imitation map $l(i): \mathcal{N} \rightarrow \mathcal{N}$,

$$
l(i)=\left\{m \in \mathcal{V}(i) \cup\{i\}, \quad \text { such that } \Pi_{m}=\max _{j \in \mathcal{V}(i)}\left(\Pi_{j}, \Pi_{i}\right)\right\}
$$

which points to the agent's $i$ neighbor with the highest payoff. If there is more than one neighbor with the same maximum payoff other than agent $i$ itself, then one is picked randomly. If agent $i$ has the maximum payoff then $l(i)=i$. In the remainder of the paper we will say that agent $i$ imitates agent $j$ if $j=l(i)$. The discrete time $t$ evolution for the imitation rule becomes

$$
s_{i}(t+1)=s_{l(i)}(t) .
$$

Agent $i$ is said to be satisfied when $l(i)=i$; otherwise it will be unsatisfied and a neighbor's strategy will be imitated.

A very simple strategy update was chosen in order to compare with previous results obtained by several authors on nonadaptive networks [12-17]. In the literature other learning rules were explored. In Ref. [24] the authors study a coordination game with local interactions, where a majority local rule seems better motivated (a sort of local best reply model [25]), in order to study the selection of an equilibrium. Also Ref. [26] discusses an evolutionary process where the learning rule itself reproduces and mutates, in order to find the best learning rule for local interacting agents.

The specification of how each agent adapts its neighbors is based on a discussion in Ref. [4]. The first step in the evolution procedure takes into account a local satisfaction measure. We only allow unsatisfied agents $[i \neq l(i)]$ to change their neighborhoods. The second step involves a classification of neighbors depending on the expected payoff. An agent will have an incentive to maintain an interaction with a neighbor if the payoff received matches the possible maximum (given the strategy played); otherwise the agent will consider to dismiss the interaction. An application of this argument to the three possible pairwise interactions is straightforward. Two $C$ agents connected by a link will on each side try to maintain the interaction, because the payoff obtained is the highest allowed by its strategy. On the contrary, two $D$ agents connected by a link receive the minimum payoff allowed by its strategy, so each agent will try to dismiss this interaction. A $C$ agent and a $D$ agent connected by a link will have opposite reactions. On one hand, the $C$ agent will try to dismiss the interaction, while on the other the $D$ agent will try to maintain it. For the sake of simplicity, we assume that this opposite reaction balances. Thus the dissatisfaction experienced by a $C$ agent of being exploited by $a$ defector offsets the satisfaction of a $D$ agent for exploiting $a$ $C$ neighbor. In conclusion, given this assumption only unsatisfied $D$ agents change their interactions with $D$ neighbors.

To complete the network adaptation, we assume that whenever an unsatisfied $D$ agent dismisses a $D$ neighbor, it randomly selects a new agent from the whole network $\mathcal{N}$ and engages in a new interaction. The selected agent always accepts the new neighbor, because a priori the unknown new neighbor can improve its aggregate utility. Notice that $D$ neighbors do not contribute to the aggregate payoff of $C$ agents. Therefore cooperators are insensitive to the number of $D$ agents they have or receive.

It is worth noting that the network adaption follows the spirit of the PD game, which highlights the conflict between the individual interest and the interest of the group. While the individual interest (of the defectors) is "enhanced" by allowing $D$ agents to improve their payoff by escaping from their $D$ neighbors, the "interest of the group" is affected because they can survive attached to the cooperators.

The network adaptation is defined as a stochastic process by introducing a new parameter $p \in[0,1]$ which corresponds to the probability for an unsatisfied $D$ agent to dismiss and find a new neighbor and introduces a time scale for the network evolution. In summary, the network adaptation stage considered in this paper is as follows.

Step (iii): Each agent $i$ revisits its current neighborhood if unsatisfied $[i \neq l(i)]$, by the following network dynamics (ND): if $i$ is a $D$ agent then breaks the link with each $D$ neighbor $k_{1} \in \mathcal{V}(i)$ with probability $p$, and replace it by randomly choosing agent $k_{2}$ uniformly from the network $\mathcal{N}$. We do not allow for self-links nor for multiple links to one agent.

This rule will adapt the degree for each agent. We have that

$$
\begin{gathered}
K_{k_{1}}(t+1)=K_{k_{1}}(t)-1, \\
K_{k_{2}}(t+1)=K_{k_{2}}(t)+1
\end{gathered}
$$

while the degree of $i$ remains invariant,

$$
K_{i}(t+1)=K_{i}(t),
$$

and only the set $\mathcal{V}(i)$ changes by replacing $k_{1} \rightarrow k_{2}$. It is clear that this rule conserves the total number of links, and thus the average degree $K$.

On the one hand, the above definition of ND allows unsatisfied $D$ agents to adapt their local neighborhoods ( $p$ $>0)$ as long as they have $D$ neighbors. The adaptation allows the agents to search other $C$ agents from whom to exploit and increase their payoff. On the other hand, $C$ agents are conservative in nature, and as discussed above, they do not have an incentive a priori to endogenously adapt their neighbors: they may only accept new neighbors. Thus the network update considered establishes an asymmetric behavior as long as $p>0$.

The network adaptation depends on the parameter relation of the PD payoff. An extension of this adaptive network rule to other two-player games is straightforward. For example, a 
coordination game with strategies $\left\{e_{1}, e_{2}\right\}$ has strict Nash equilibria $\left(e_{1}, e_{1}\right)$ or $\left(e_{2}, e_{2}\right)$. Unsatisfied agents imitating opponents with opposite strategy [either $\left(e_{1}, e_{2}\right)$ or $\left.\left(e_{2}, e_{1}\right)\right]$ would prefer to change partners.

Finally, we remark that unlike other studies in adaptive networks, we do not allow an indirect payoff due to the neighbors' neighbors, as for example in Refs. [10,27]. Therefore we analyze the evolution based on strict nearest neighbors' interactions.

\section{ANALYSIS}

In this section we provide results on the organization and stability of the network, by having a close look to the steady state solutions. These states arise in a dynamical evolution of the game whenever $p>0$, and correspond to network configurations and individual strategies which remain stationary in time. The multiplicity of these solutions is huge, for a fair number of agents. However, they have certain common features that we now reveal. Also, and very interesting, perturbations acting on them may produce significant changes to the whole network. The more complicated dynamical aspects of evolution will be dealt with in Sec. IV, where numerical simulations will be helpful. Also in this section, we introduce the notion of leaders, which is important in order to understand how the asymptotic steady state may be reached: either a nearly full cooperative state, or a full defective one. These results will be exemplified in Sec. IV.

\section{A. Existence of steady states}

As defined above, steady states will refer to equilibrium states where the network configurations and individual strategies remain stationary in time. For the sake of clarity, let us first consider some simple examples. Consider the steady state in the nonempty $(K \neq 0)$ adaptive $(p>0)$ network composed by only $C$ agents. In this case, agents are either satisfied if they have the maximum payoff of their neighborhood, i.e., $l(i)=i$, or otherwise conformists (if they are unsatisfied) because they imitate their same strategy from a better agent. In both cases, they will not change their individual payoff, nor their local neighborhoods, for there are no $D$ agents in network $\mathcal{N}$. In the other extreme, for a network composed of only $D$ agents, the average payoff remains stationary in time, but the network configuration and the individual payoff will be perpetually evolving - unless they all have the same number of neighbors so that everyone is satisfied, and ND does not apply; a very unlikely situation we do not consider. From the above definition, we will not consider the latter example a steady state, and we refer to these dynamic configurations without cooperators as the all-D network. It is worth noting that this set of configurations is absorbing because once the system reaches this state, the dynamics itself cannot escape from it. This state is of interest for it is one of the possible asymptotic states the dynamics of the system may reach.

Let us introduce the definition of chain of agents. Due to the strategy update we implemented, forward iteration of the imitation map $l(i)$ starting from any agent $i$ traces a connected component in the network configuration with nonde-

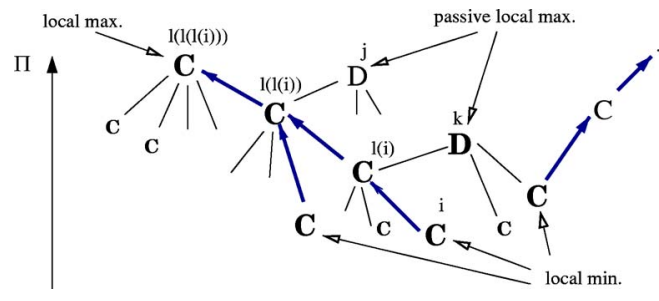

FIG. 1. (Color online) Schematic diagram of several chains of cooperators. The vertical axis corresponds to the total individual payoff $\Pi_{i}$. The thick arrows represent the imitation map [Eq. (3)]. Agent $l^{3}(i)$ is a local maximum, $D$ agents $j$ and $k$ are passive local maxima, while several local minima are also shown.

creasing payoff. Thus a chain of agents starting at agent $k$ and length $m$ is defined as the subset $\left\{k, l(k), \ldots, l^{m-2}(k), l^{m-1}(k)\right\} \in \mathcal{N}$, where $\Pi_{l^{r}}>\Pi_{l^{s}}$ with $m-1$ $\geqslant r>s \geqslant 0$, and ends whenever agent $l^{m-1}(k)$ is satisfied $\left[l^{m}(k)=l^{m-1}(k)\right]$. In particular we will discuss the behavior of chains of cooperators and defectors.

Let us define a local maximum in payoff as the agent $i$ with all its neighbors $j \in \mathcal{V}(i)$ satisfying $\Pi_{i}>\Pi_{j}$ and at least a neighbor $j$ imitates the local maximum, $i=l(j)$. However, we distinguish as a passive local maximum the extreme situation where none of the neighbors $j$ imitate agent $i: \forall j$ $\in \mathcal{V}(i), i \neq l(j)$ and $\Pi_{l(j)}>\Pi_{i}>\Pi_{j}$. Finally a local minimum in payoff corresponds to an agent $i$ such that all neighbors $j$ satisfy $\Pi_{i}<\Pi_{l(j)}$. Figure 1 illustrates several chains of cooperators, ordered vertically by their corresponding individual payoff of the agent. For example, the chain $\left\{i, l(i), l^{2}(i), l^{3}(i)\right\}$ has attached also two passive local maxima (agent $j$ and $k$ ).

In general in an adaptive network where $p>0$, nontrivial steady states may arise. Chains of cooperators are a possible stable situation, for ND does not apply. On the other hand, a chain of defectors is unstable, for ND applies on all its members except the local maxima. Thus in steady states configurations defectors cannot form chains, and may only have $C$ neighbors. More specifically $D$ agents must be passive local maxima in order for their strategy not to be replicated by its neighbor chains of cooperators, i.e., no agent imitates $D$ agents. In conclusion, the only stationary situation in terms of strategy and network configurations leading to steady states involves $C$ agents forming chains, where replication of $C$ strategy takes place, while $D$ neighbors do not form chains and are passive local maxima. In particular $D$ agents may become isolated or ostracized (without links) [28].

It is clear that for a fair number of agents, the multiplicity of chains is huge. However, only those chains of cooperators which satisfy a stability criterion will survive. Denote $K_{i}^{\mathrm{CC}}$ as the number of $C$ neighbors the $C$ agent $j$ has, while $K_{j}^{\mathrm{bC}}$ is the number of $C$ neighbors the $D$ agent $j$ has. A chain of $m$ $>0$ cooperators $\left\{k=l^{0}(k), l^{1}(k), \ldots, l^{m-1}(k)\right\}$ must satisfy the following ordering:

$$
\begin{aligned}
& K_{l^{s+1}(k)}^{\mathrm{CC}}>b K_{r}^{\mathrm{DC}}+\delta K_{r}^{\mathrm{DD}} \geqslant K_{l^{s}(k)}^{\mathrm{CC}}, \\
& \forall s \in[0, m-1], \text { and } r \in \mathcal{V}\left(l^{s}(k)\right)
\end{aligned}
$$



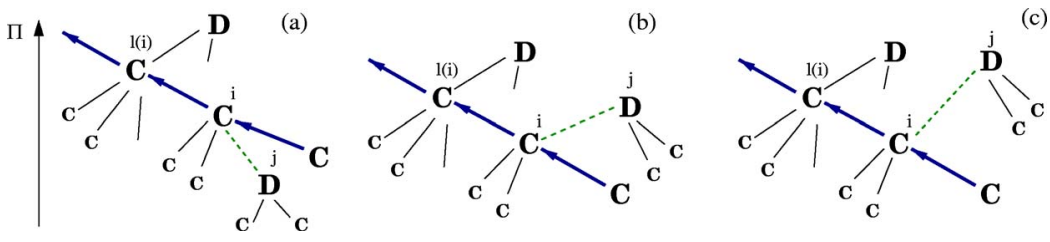

FIG. 2. (Color online) Schematic diagram showing different types of perturbations to a chain of cooperators $\xi$ (see text). By the ND, the $D$ agent $j$ chooses as a new neighbor agent $i$ establishing a new link (dashed line) at time $t$. The diagram illustrates the payoffs computed at $t+1$ after performing the stage [step (i)] and before [step (ii)]: (a) $\Pi_{i}(t+1)>\Pi_{j}(t+1)$, constructive perturbation, (b) $\Pi_{l(i)}(t+1)>\Pi_{j}(t+1)$ $>\Pi_{i}(t+1)$, neutral perturbation, and (c) $\Pi_{j}(t+1)>\Pi_{l(i)}(t+1)>\Pi_{i}(t+1)$ destructive perturbation. The arrows represent the imitation map.

in order to be a stationary configuration.

In conclusion we have the following:

Proposition 1 . In the adaptive game $(p>0)$, a steady state exists only when all $D$ agents are either passive local maxima which interact exclusively to $C$ agents in a chain satisfying Eq. (8), or are isolated (have no links).

In other words, a steady state is composed exclusively by a subnetwork composed of the chains of cooperators, together with the subnetwork of defectors only exploiting $C$ neighbors. In a dynamical evolution for $0<p \ll 1$, the imitation dynamics is much faster than the network evolution, and strategies equilibrate faster than the neighborhoods. Therefore a useful indicator to control whether the system reached a steady state for the numeric simulations in Sec. IV is to monitor when the number of links between $D$ agents vanishes.

Also notice that the result of the previous proposition holds even for extremely small $p>0$, which shows a clear distinction to the steady states which might occur for $p=0$. In general, fixed neighborhoods of $D$ agents with more than one $D$ agent are possible, in clear contrast to our results for $p$ $>0$, where $D$ agents only interact with $C$ agents.

\section{B. Stability of steady states}

Once we have established the properties of the steady states, we investigate their stability. We will show that particular perturbations will grow cooperative networks, while some others may trigger large scale dynamics in the whole network.

Consider a steady state decomposed in a collection of chains of cooperators $\left\{\xi_{j}\right\}$. Stability can now be studied on each component $\xi_{j}$. Let us assume the network is "close" to a steady state, where the total number of links between $D$ agents is close to zero, and most chains $\xi_{j}$ are in equilibrium satisfying Eq. (8). By the ND, the remaining clusters of $D$ agents will change their $D$ neighbors repeatedly "searching" for $C$ neighbors. These perturbations will affect not only the $D$ agents' own payoff, but may produce changes in the local neighborhood of the new chosen agent. Different situations may arise depending on the actual payoff of the perturbing $D$ agent, and in which $C$ agent the perturbation falls.

In Fig. 2 we show a schematic diagram showing three different cases which may arise. Consider a chain of cooperators $\xi$ receives the new $D$ neighbor $j$ at time step $t$ after performing the stage [step (iii)]. On time step $t+1$ the payoffs $\Pi_{i}, \Pi_{j}$, and $\Pi_{l(i)}$ are again computed at stage [step (i)] and their relative value may fall in any of the possibilities shown in Fig. 2 [29]:

(a) The first situation corresponds to the payoff relation $\Pi_{j}<\Pi_{i}$ [see Fig. 2(a)]. If $l(j)=i$, then at the end of this time step the perturbation $D$ agent will become a $C$ agent by imitation. However, if $l(j)$ points to another $D$ agent, then we may guarantee only that there is a finite probability that the $D$ perturbation becomes a $C$ agent. This occurs when in a number of time steps (depending on $p$ ) ND applies, and $D$ agent $j$ changes $l(j)$ by a new neighbor which may either have a lower payoff than $j[$ so that $i=l(j)$ in the next time step], or the new neighbor is a $C$ agent with a larger payoff than $j$. In both cases, agent $j$ imitates $C$ strategy in the following time step. Of course, during these intermediate time steps the network also evolves, and again we can only conclude a finite probability for the switch of strategy. In conclusion depending on the neighborhood structure of the $D$ perturbation $j$, there is a finite probability that $j$ becomes a $C$ agent. We will refer to this type of perturbations as constructive, and is a primary mechanism to enlarge the cooperator's population.

(b) The second situation corresponds to the payoff relation $\Pi_{l(i)}>\Pi_{j}>\Pi_{i}$ [see Fig. 2(b)]. By a similar argument as in the previous case, depending on the composition of $\mathcal{V}(j)$ there is a finite probability that $D$ agent $j$ becomes a passive local maximum in a number of steps, so we will refer to these perturbations as a neutral perturbation. If at time step $t+1, l(j)=j$, then with probability one the perturbation $j$ becomes a passive local maximum to $j$. This is a primary mechanism where $D$ agents may survive, with a continual exploitation to $C$ agents.

(c) The third situation corresponds to the payoff relation $\Pi_{j}>\Pi_{l(i)}>\Pi_{i}$ [see Fig. 2(c)]. This corresponds to a destructive perturbation, for $D$ strategy is replicated "downhill" (in payoff) through the chains composed of $\xi^{\prime}=\{i, u, v, \ldots\}$, where $u, v, \ldots$ are such that $l(u)=i, l(v)=u, \ldots$. By this mechanism one expects an increase in the defector's population.

We have established the following:

Proposition 2. Given an adaptive game $(p>0)$, a perturbation at time $t$ of a $D$ agent $j$ to a $C$ agent $i$ belonging to a chain $\xi \equiv\left\{\ldots, i, l(i), l^{2}(i), \ldots\right\}$ containing at least two cooperators can generate different outcomes depending on the payoff relation between $\Pi_{j}(t+1), \Pi_{l(i)}(t+1)$, and $\Pi_{i}(t+1)$.

(a) Constructive perturbation: If $\Pi_{i}(t+1)>\Pi_{j}(t+1)$ there is a finite probability that in the subsequent time steps the chain of cooperators enlarges by incorporating $j$. The probability is one if $l(j)=i$ at time step $t+1$. 

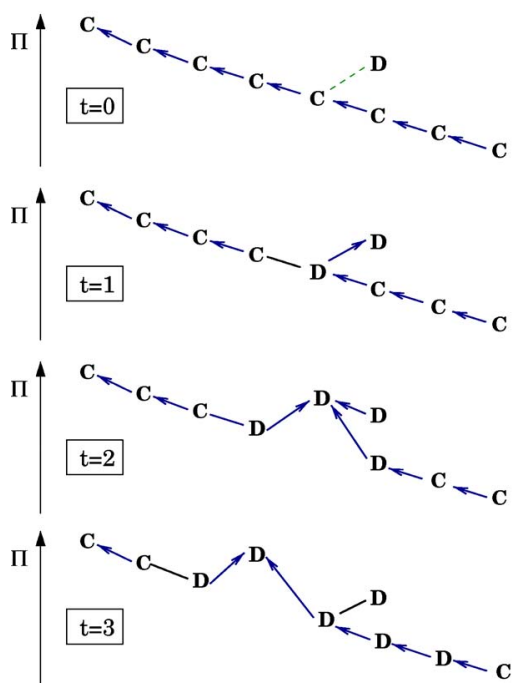

FIG. 3. (Color online) Schematic diagram showing the evolution of an uphill destructive cascade. At time $t=0$ a $D$ agent selects a $C$ agent as a new neighbor. The subsequent time steps show how the replication of $D$ strategy not only goes downhill in payoff function, but also uphill. The latter perturbations are allowed if the payoff gap between neighbor $C$ agents have are below a specific bound [see Eq. (13)]. The arrows represent the imitation map.

(b) Neutral perturbation: If $\Pi_{l(i)}(t+1)>\Pi_{j}(t+1)$ $>\Pi_{i}(t+1)$ there is a finite probability that in the subsequent time steps $D$ agent $j$ becomes a passive local maximum to $i$. The probability is one if $l(j)=j$ at time step $t+1$.

(c) Destructive perturbation: If $\Pi_{j}(t+1)>\Pi_{l(i)}(t+1)$ $>\Pi_{i}(t+1)$ then with probability one replication of $D$ strategy starts over a subset of the original chain $\{i, u, v \ldots\} \in \xi$ (where $u, v, \ldots$ are such that $l(u)=i, l(v)=u, \ldots$ ), enlarging the defector's population.

A very interesting recursive property may appear in the destructive perturbations above. Let us denote agent $k=l(i)$ at time step $t$. At time step $t+2$, agent $i$ displays a considerable payoff jump, $\Pi_{i}(t+2) \geqslant b \Pi_{i}(t+1)>\Pi_{i}(t+1)$, and must be considered a new perturbation to agent $k$. From Proposition 2 it depends on the payoff of $\Pi_{l(k)}(t+2), \Pi_{k}(t+2), \Pi_{i}(t$ $+2)$, a constructive, neutral, or destructive perturbation may occur. If a constructive perturbation results for time step $t$ +3 , then part of the "downhill" destructive perturbations may be recovered into $C$ agents from $\xi$ '. If a neutral perturbation results, then agent $i$ becomes a passive local maximum, and the original chain $\xi$ ends at the new local minimum at agent $k$. Finally if agent $i$ becomes a new destructive perturbation to $l(k)$, then this would have accomplished the interesting feature that destructive perturbations may also travel "uphill" in payoff function. The above dynamical process is illustrated in Fig. 3, where a schematic simulation is shown (together with the downhill replication process) and a sequence of uphill perturbations reach the local maximum and completely destroy the original chain of cooperators. Below we study conditions under which these perturbations may occur and are found to be very important in the development of cooperative steady states.

\section{Leaders' evolution}

The network adaptation shapes the steady state network in a very heterogenous manner. We define a $C$ leader $(D$ leader), as the $C$ agent ( $D$ agent) with the largest payoff among the $C$ agents ( $D$ agents). Out of all the $C$ agents, the chains local maxima are candidates to be the $C$ leader. Like- wise for $D$ agents, among all the passive local maxima, there will be one with the maximum payoff and it will be the $D$ leader. We now describe an important property leaders share, responsible for the asymptotic network evolution.

Let us denote by $\alpha(\beta)$ the $C(D)$ agent with the maximum payoff in the whole system. Due to the "searching" done by unsatisfied $D$ agents, it is clear that if the leaders' payoff satisfies $\Pi_{\alpha}>\Pi_{\beta}+b$, then any link received by leader $\alpha$ from any $D$ agent will be necessarily of constructive type, following Proposition 2(a). Then, for each perturbation received by the leader on time step $t$, the leaders' payoff evolves on the next time step as

$$
\Pi_{\alpha}(t+1)=\Pi_{\alpha}(t)+1, \quad \Pi_{\alpha}>\Pi_{\beta}+b .
$$

In fact, this result also holds for all $C$ agents $i$ whose payoffs satisfy $\Pi_{i}>\Pi_{\beta}+b$. We will refer to this subset of $C$ agents as "wealthy agents," and provided the condition is satisfied, its payoff (and degree) is always nondecreasing in time.

However, an unstable situation occurs whenever the $C$ leader does not have the largest payoff in the whole network. In this case the leader $\alpha$ has a finite probability of receiving a $D$ neighbor with a larger payoff, which will produce a drastic event in the subsequent time steps: $\alpha$ and its associated chains will replicate the $D$ strategy. As an extreme situation consider $C$ leader $\alpha$ leading a number of chains of cooperators, and the rest of the population consists of $D$ agents. We can easily find a lower bound on $\Pi_{\alpha}$ such that the other $C$ agents have a chance of survival:

$$
\Pi_{\alpha}>\Pi_{\gamma}+b
$$

where $\gamma$ is the $D$ agent with minimum payoff among defectors. Otherwise all perturbations the $C$ agents receive from the $D$ agents will be destructive. In this case, depending on the actual configuration of the chain and the perturbations received, the evolution of the network has a large probability of reaching the all- $D$ network.

The above discussion establishes two bounds on the payoff of $C$ leaders for their development and survival,

Proposition 3. Let us consider $\alpha(\beta)$ as the cooperator (defector) with the largest payoff among all cooperators (defec- 
tors) and $\gamma$ as the defector with the lowest payoff among defectors. If $p>0$ and the network is not in a steady state then:

(a) If $\Pi_{\alpha}(t)>\Pi_{\beta}(t)+b$, each perturbation received by $\alpha$ at time step $t$ will be of constructive type and increases the leader's payoff: $\Pi_{\alpha}(t+1)=\Pi_{\alpha}(t)+1$. The same result holds for all $C$ agents $i$, labeled "wealthy agents," such that $\Pi_{i}$ $>\Pi_{\beta}+b$.

(b) If $\Pi_{\gamma}(t)>\Pi_{\alpha}(t)$, perturbations received by all $C$ agents at time $t$ will be destructive, and drive the system towards the absorbing all- $D$ network.

One important consequence of Proposition 3(a) is that it provides conditions such that cooperative leaders emerge in the dynamical evolution of the proposed game. In other words the payoff of wealthy agents does not follow a random stochastic process; on the contrary, they have a nondecreasing payoff in time provided the condition on leaders' payoff is satisfied. Another consequence of this result is that the wealthy agents are constantly absorbing perturbations of unsatisfied $D$ agents and thus helping the network approach a steady state.

\section{Example: Full capacity cooperators chain}

In the previous section we studied the dynamics of leaders; we now turn to a tractable example for the dynamics of a chain. Consider a chain $\xi=\{1, \ldots, i, i+1, \ldots m\}$ [where for simplicity we have renamed the agents such that $l(i)=i+1$, $\forall i \neq m]$ which is said to be with "full capacity," i.e., each $C$ agent $i \neq m$ (except the leader) in the chain satisfies $K_{l(i)}^{\mathrm{CC}}$ $=K_{i}^{\mathrm{CC}}+1$. Let us assume that all chain members (except the absolute maximum $m$ ) have exactly $s$ passive local maxima $D$ agents (exploiters), and denote by $r=K_{i}^{\mathrm{CC}}$ the number of $C$ neighbors the $C$ agent $i$ has. We can now study in some detail how after a destructive perturbation acts on agent $i$, a new constructive, passive, or destructive perturbation may be produced as a function of the parameters $(b, \delta)$, and the integer pair $(r, s)$.

Consider that at time $t$ any of the $s$ exploiters of $i$ receives a perturbation such that Proposition 2(c) holds. At time $t$ +1 , agent $i$ becomes a $D$ agent with a payoff $\Pi_{i}(t+1)=b r$ $+\delta s$. Now the condition that $i$ becomes a new passive local maximum to agent $i+1=l(i)$ is $r+2>\Pi_{i}(t+1)>r+1$. This is true for the interval of $b$ defined by

$$
b_{+}^{r, s} \equiv 1+\frac{2-\delta s}{r}>b>1+\frac{1-\delta s}{r} \equiv b_{-}^{r, s} .
$$

In Fig. 4(a) we show the locus of $b_{ \pm}^{r, s}$ as a function of $\delta$ for different pairs of integers $(r, s)$. The region in between the parallel lines corresponds to where Eq. (11) is satisfied, and therefore the perturbation generates a new passive local maximum (P in Fig. 4). Below this region, we have constructive perturbations (denoted by $\mathrm{C}$ ), where after Proposition 2(a) agent $i$ becomes again a $C$ agent after some finite steps. Above the region $\mathrm{P}$, we have destructive perturbations ( $\mathrm{D}$ in Fig. 4), where the $D$ agent $i$ becomes the best neighbor of $i+1$, thus becomes a perturbation satisfying again Proposition 2(c). Then on the next time step (and for the special
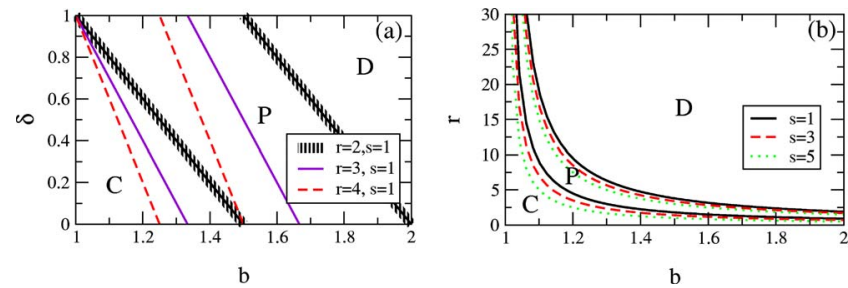

FIG. 4. (Color online) Stability diagram for local perturbations: constructive (C), passive (P), and destructive (D) perturbations. (a) Locus of $b_{1,2}^{r, s}$ in the PD parameter space $(b, \delta)$. (b) Locus of $r_{1,2}(b, \delta, s)$ in terms of incentive to defect $b$. The curve corresponding to $s=0$ falls very close to $s=1 \quad(\delta=0.1)$.

assumption of a chain in full capacity), agent $i+1$ satisfies the condition of a destructive perturbation on agent $i+2$, and so on until the chain is completely destroyed.

Another alternative scenario occurs if the original perturbation on $i$ is passive, for if $s>0$ the new chain is not in a steady state. The "search" done by $i$ and its $s D$ neighbors will gradually improve the payoff of $i$, until it becomes a destructive perturbation to $i+1$. This is true when $(r+2) /(r$ $+s)>b>(r+1) /(r+s)$; for $s>1$ this cannot happen in a PD game as defined above, with $1<b<2$.

Another way to illustrate how vulnerable are full capacity chains is to fix $\delta$ and $s$, and study from Eq. (11) the possible $r$ 's which give passive perturbations,

$$
r_{+}(b, \delta, s) \equiv \frac{2-\delta s}{b-1}>r>\frac{1-\delta s}{b-1} \equiv r_{-}(b, \delta, s) .
$$

Figure 4(b) shows the dependence of $r_{+}(b, \delta, s)$ as $b$ is increased, and shows how vulnerable to destructive perturbations "full capacity" chains are.

\section{E. Payoff gaps and protection to leaders}

The previous example shows that chains with a small payoff difference between consecutive agents in the chain [e.g., $i$ and $l(i)]$, are easily wiped out by uphill perturbations. In order for steady states to become more robust to these perturbations, the chain must develop "payoff gaps," where $\Pi_{l^{2}(i)} \gg \Pi_{i}$. More precisely, consider that a perturbation reaches agent $i$. In order for an uphill perturbation not to develop the payoffs must satisfy

$$
\Pi_{l^{2}(i)}>b \Pi_{i}+\delta K_{i}^{\mathrm{CD}}
$$

before receiving the perturbation. For the purpose of illustration we show in Table I examples of "minimal" chains which satisfy Eq. (13) and uphill perturbations are inhibited, where we have assumed a fixed number of passive local maxima $s \equiv K^{\mathrm{CD}}=3$, and different values of $b$. The values were obtained by iterating the function $f(i+1)=\lceil b / ; i+\delta s\rceil$ (where $\lceil x\rceil$ is the largest integer greater than $x$ ). Clearly these minimal chains which do not allow uphill perturbations have payoff gaps which increase for larger $b$. Thus an important conclusion is that in a dynamic evolution one expects uphill perturbations to occur more frequently for larger $b$.

The above characterization is important because we may now distinguish those "dangerous chains" such that their 
TABLE I. Chains of cooperators with gaps that inhibit uphill perturbations. For this example we took $\delta=0.1$, and a fixed value of $s \equiv K^{\mathrm{CD}}=3$ passive local maximum.

\begin{tabular}{cccccc}
\hline \hline$b$ & $K_{i}^{\mathrm{CC}}$ & $K_{i+2}^{\mathrm{CC}}$ & $K_{i+4}^{\mathrm{CC}}$ & $K_{i+6}^{\mathrm{CC}}$ & $K_{i+8}^{\mathrm{CC}}$ \\
\hline 1.55 & 4 & 7 & 11 & 17 & 26 \\
1.55 & 5 & 9 & 13 & 21 & 32 \\
1.55 & 6 & 10 & 16 & 24 & 38 \\
1.75 & 5 & 10 & 17 & 29 & 51 \\
1.95 & 5 & 11 & 20 & 40 & 77 \\
\hline \hline
\end{tabular}

payoff gaps do not inhibit uphill cascades and reach the local maximum or $C$ leader. A $C$ leader is "protected" when all its associated chains are not dangerous. Notice that whenever the $C$ leader replicates the $D$ strategy, a large multiplicative effect occurs, for all the chains connected to the $C$ leader will replicate by downhill perturbations the $D$ strategy. It is clear that this will give origin to a large cascade affecting large portions of the whole network (see Sec. IV E).

A closer analysis of the network dynamics reveals a mechanism which reduces the number of dangerous chains and provides protection to the $C$ leader (and possibly $C$ local maxima). Given the payoff of $D$ leader $\beta$, on each chain in the $C$ cluster let us focus on the particular agent $i^{*}$ such that the payoffs of agents $l\left(i^{*}\right)$ and $l^{2}\left(i^{*}\right)$ satisfy

$$
\Pi_{l^{2}\left(i^{*}\right)}>\Pi_{\beta}>\Pi_{l\left(i^{*}\right)}
$$

Consider only perturbations which arise from unsatisfied $D$ agents which do not belong to the $C$ cluster, i.e., these perturbations which do not arise from an uphill process. It is straightforward to show using Proposition 2 that agent $l\left(i^{*}\right)$ may only receive neutral perturbations, while for $l^{2}\left(i^{*}\right)$ the perturbations are only of the constructive type. Thus the payoff gap $\Omega\left(l\left(i^{*}\right)\right)=\Pi_{l^{2}\left(i^{*}\right)}-\Pi_{l\left(i^{*}\right)}$ will be nondecreasing in time for this payoff of agent $\beta$. Note that the payoff of $\beta$ fluctuates in time, thus this effect will increase payoff gaps of different pairs of agents. The precise condition for a chain to become immune to an uphill cascade corresponds to Eq. (13) with $i=i^{*}$.

On the other hand, if the chain is not protected and an uphill cascade develops, then on the next time step there is a finite probability that a new uphill perturbation occurs. In particular agent $i^{*}$ becomes the new $D$ leader and a new $i^{*}$ agent must be selected on each chain of cooperators. In the numerical examples in Sec. IV E we will show this process in action. We summarize these results in the following:

Proposition 4. Suppose there exists a $C$ local maximum $\alpha$ with a cluster of agents forming chains of cooperators. Suppose that the $D$ leader $\beta$ satisfies $\Pi_{\alpha}>\Pi_{\beta}+b$, then for each chain in the cluster there exist two agents $i^{*}$ and $l\left(i^{*}\right)$ which satisfy Eq. (14). Then the payoff gap of the chain $\Omega\left(l\left(i^{*}\right)\right)$ $=\Pi_{l^{2}\left(i^{*}\right)}-\Pi_{l\left(i^{*}\right)}$ is a nondecreasing function in time, whenever the payoffs of agents $i^{*}$ and $l^{2}\left(i^{*}\right)$ satisfies Eq. (13). Under these conditions the mechanism effectively reduces the number of dangerous chains in the cluster by 1 .
The application of the above result to each dangerous chain connected to the $C$ leader may effectively protect the $C$ leader from uphill perturbations. A consequence of this result is that only when all $C$ local maxima in the network are protected the network will reach surely a cooperative steady state, for there will be an invariant number of wealthy $C$ agents $\Pi_{i}>\Pi_{\beta}+b$ which will absorb continuously constructive perturbations and drain the total number of perturbations. Clearly, the above statement provides sufficient conditions such that a steady state may be reached; in computer simulations a steady state may still have dangerous chains which have not been exploited by uphill perturbations.

\section{NUMERICAL SIMULATIONS}

We will now address by the use of computer simulations how the dynamic model evolves in time, and the central question of how cooperation performs in such an adaptive environment. Our results show that cooperation emerges as a steady state for the whole range of $1<b<2$ and all networks studied. Also, surprisingly, the average payoff of defectors is larger than that of cooperators. This points to the existence of leaders, which have a very important role in determining the asymptotic evolution in time of the system. The main parameters in this investigation will be the incentive to defect $b$, the network adaptation rate $p$, and the average degree of the network $K$.

The statistical measures we studied have been

(i) the fraction of cooperating agents ( $C$ agents), denoted $f_{\mathrm{C}}=\left(\sum_{i=1}^{N} s_{i}\right) / N$;

(ii) the probability of having a link between two $C$ agents, $p_{\mathrm{CC}}$, between a $C$ agent and a $D$ agent, $p_{\mathrm{CD}}$, and between two $D$ agents, $p_{\mathrm{DD}}$. These quantities satisfy

$$
1=p_{\mathrm{CC}}+2 p_{\mathrm{CD}}+p_{\mathrm{DD}} \text {. }
$$

Simulations were performed with a total number of agents in the range $N \in[300,10000]$, displaying qualitatively the same results. We have adopted $N=10000$ as the base case. With this number of agents, the initial fraction of randomly distributed $C$ agents in the network $\mathcal{N}$ was set to $0.6 N$. Any initial fraction of $C$ agents above this value was observed not to influence the final outcome. Clearly, if the randomly distributed $C$ agents does not form a sufficiently wealthy $C$ leader, cooperation may not be promoted and the final state is an all- $D$ network (see Sec. III C). Also all time series have been evolved during an initial 50 time steps without network adaptation (nor indicator measurements), to allow for a transient behavior due to the random initial distribution of strategies. The starting network is random: $K N / 2$ links are distributed randomly among the $N$ agents in the network.

\section{A. Temporal evolution and steady states characteristics}

A typical time evolution of cooperation fraction $f_{\mathrm{C}}$ is displayed in Fig. 5(a). First notice that the cooperative fraction increases on average steadily, until most of the network agents are cooperative. Computation of the density of all the possible links among agents reveals that the number of links 

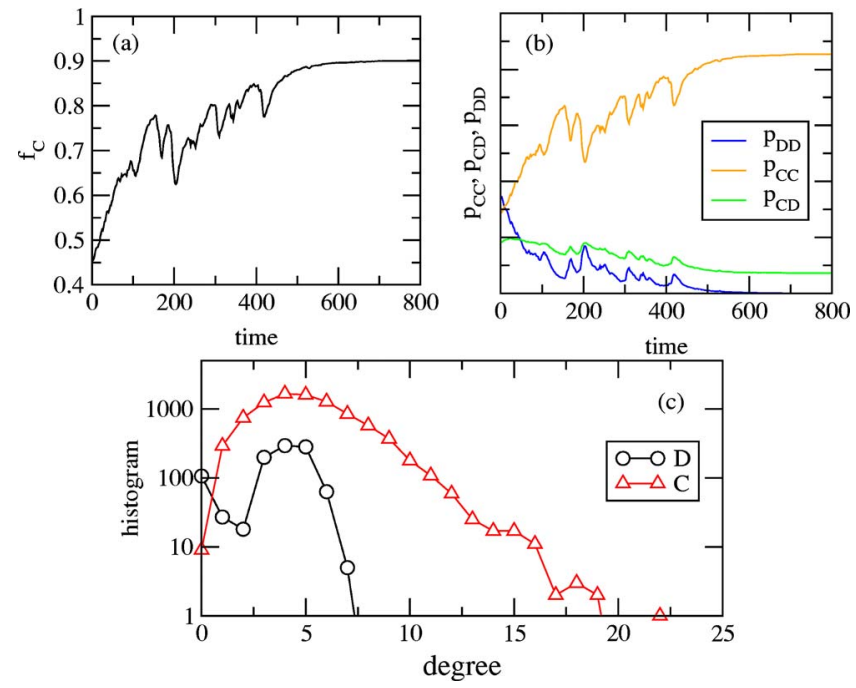

FIG. 5. (Color online) Time evolution of (a) the fraction of cooperators $f_{\mathrm{C}}$, and (b) the probability to have a link between two $C$ agents $\left(p_{\mathrm{CC}}\right)$, a $C$ and a $D$ agent $\left(p_{\mathrm{CD}}\right)$, and two $D$ agents $\left(p_{\mathrm{DD}}\right)$. (c) Degree distribution for cooperators and defectors in the steady state. Parameter values: $p=0.015, b=1.45$, and $K=5$.

between $D$ agents, decreases also on average steadily. The steady state is reached at $T=700$ when there are no links between $D$ agents $\left(p_{\mathrm{DD}}=0\right)$ and the chains of cooperators satisfy Eq. (8), as Proposition 1(a) shows. On the other hand the number of links between $C$ and $D$ agents is low (most of the time below 20\%). Therefore the structure of the steady state network is composed by chains of cooperators together with exploiting $D$ agents.

A closer look at the distributions of links corresponding to the steady state is shown in Fig. 5(c), which reveals differences in how $C$ and $D$ agents connect. In general one obtains an exponential decrease in the number of links between $C$ agents with $C$ agents ( $C$ - $C$ links), while $D$ agents exploit a narrow distribution of $C$ agents. Notice also that the maximum number of links $C$ - $C$ links may become several times the average degree $K$, and there is approximately $10 \%$ of solitary $D$ agents without links at all.

Extensive numerical simulations reveal that there are two types of temporal evolutions in our model. For approximately $b<1.45$ the evolution is characterized by a roughly steady increase in $f_{\mathrm{C}}$ [as shown in Fig. 5(a)], a relatively constant $p_{\mathrm{CD}}$, while $p_{\mathrm{DD}}$ decreases on average monotonously until complete extinction. On the other hand, when $b>1.45$ some realizations display global oscillations in the fraction of cooperators. In this case, $p_{\mathrm{CD}}, p_{\mathrm{CC}}$, and $p_{\mathrm{DD}}$ are coupled together, and follow always the trend displayed in Fig. 6. The figure shows a typical time evolution where several large global cascades developed before settling into a cooperative steady state. The number of large cascades may vary significantly from run to run. The final outcome of the above temporal evolution may either be a cooperative steady state, or the all- $D$ network. The origin of these large cascades and the asymptotic outcome relies on a strong interaction between $C$ and $D$ leaders, and a detailed discussion is delayed until Sec. IV E.

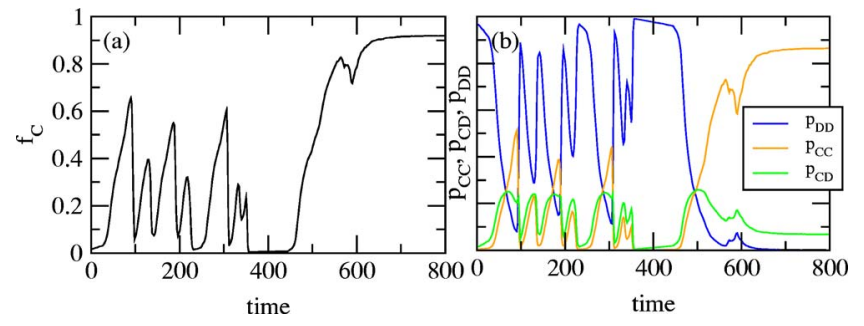

FIG. 6. (Color online) Time evolution of (a) the fraction of cooperative agents $f_{\mathrm{C}}$, and (b) the probability to have a link between two $C$ agents $\left(p_{\mathrm{CC}}\right)$, a $C$ and a $D$ agent $\left(p_{\mathrm{CD}}\right)$, and two $D$ agents $\left(p_{\mathrm{DD}}\right)$. Parameter values: $p=0.03, b=1.75$, and $K=8$.

\section{B. Cooperation enhancement}

We now characterize with numerical experiments how cooperation performs asymptotically in time for various values of the incentive to defect $b$, network adaptation $p$, and average degree $K$.

Figure 7 displays the results of the fraction of $C$ agent $f_{\mathrm{C}}$ as a function of the incentive to defect $b$. The experiment was run for 50 random realizations, and a maximum time of integration of $T=1000$ time steps. Given the network adaptability $p=0.1$ chosen, the total time of integration was sufficient in order for the system to reach either a steady state, or the all- $D$ network. As before, a steady state was found whenever the links between $D$ agents disappeared $\left(p_{\mathrm{DD}}=0\right)$. To characterize solely the cooperative steady state for $p>0$, we did not include in the statistical average of Fig. 7 those realizations reaching the all- $D$ network. The $f_{\mathrm{C}}=0$ absorbing state is present in the whole range of $b$, and our results show that depending on the initial condition, the system may reach either of them.

Numerical simulations with different average degree $K$ does not change the general behavior of the fraction of cooperation. For $p=0.1$ the average cooperation is over $94 \%$ for $K=12,89 \%$ for $K=8$, and $75 \%$ for $K=4$ for $b<2$. We also tested regular lattices as initial networks, as those stud-

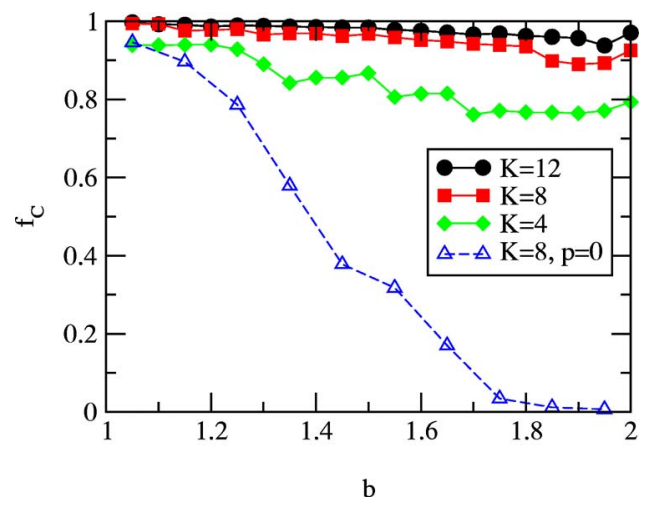

FIG. 7. (Color online) Fraction of cooperation $f_{\mathrm{C}}$ as a function of $b$ for $K=4,8,12$ and $p=1.0$. The filled symbols indicate that all the averaged realizations reached a steady state. The nonadaptive case $p=0, K=8$ (dashed line) seldomly reached a steady state, and the average was performed on all final conditions, including all- $D$ networks. The maximum integration time was $T=1000$ time steps and 50 random realizations for every point. 
ied in Ref. [15]. The resulting final network was random, and no trace of the initial regular lattice was observed, e.g., comparison of the average asymptotic value of $f_{\mathrm{C}}$ starting with a regular first neighbors lattice was statistically insignificant to that of starting from a random $K=4$ lattice. For a network adaptation $p>0.1$, cooperation was enhanced and the difference between $K=8$ and $K=12$ was reduced to become nearly identical at $p=1$.

On the other hand, for smaller values of $p$, the network adaptability occurs less frequently, and a larger integration time was required to reach a final state. Making numerical simulations with a fixed integration time $T=T^{*}$, and performing an analysis of the average value of $f_{\mathrm{C}}\left(T^{*}\right)$ as a function of $p$, shows a continuous crossover from the high cooperation level found in cooperative steady states to those found in nonadaptive networks $p=0$.

For comparison, results on a random nonadaptive networks $(p=0)$ is also included in Fig. 7. Our results are in agreement with those obtained by other authors [30]: the fraction of cooperation decreases as $b$ increases, up to a critical $b^{*}$ where defection always dominates. As long as there is a single $D$ agent, for $b>b^{*}$ the system asymptotically goes to the all- $D$ network. For $K=8$ and $\delta=0.1$ we have obtained an approximate $b^{*} \approx 1.75$.

This marks a strong contrast between adaptive and nonadaptive networks. In the latter case partial cooperation is found up to a critical $b^{*}$, after which cooperation disappears. The former case displays an enhanced cooperation throughout the whole range of $b$, which coexists with the absorbing all- $D$ network. In the case of nonadaptive network $D$ agents can get a larger profit only at the boundaries where they meet $C$ agents to exploit [15]. By enumerating the finite neighborhood configurations possible at the boundary of $C$ and $D$ agents in regular networks, one may find a lower bound on $b$ such that $D$ strategy is replicated by the boundary $C$ agents (thus the $D$ agents advances and invades a cluster of $C$ agents). With the adaptive network considered here, clusters of $D$ agents tend to dissappear and only $D$ agents exploiting a large number of $C$ agents is able to replicate $D$ strategies.

\section{Wealth distribution}

Although cooperation in steady states is highly enhanced throughout the network, we find an uneven wealth distribution among agents. The surprising result is that the average payoff of $D$ agents in a steady state is found to be larger than $C$ agents, and increases with larger $b$.

A comparison is illustrated in Fig. 8, where the wealth distribution for a low and a large value of $b$ is shown. This is a specific consequence of the selective adaptability of the network, and does not occur with fixed and regular neighborhoods as those studied in previous works $[15,32]$. The network adaptation favors $D$ agents to search for $C$ agents to exploit, until they become satisfied (or become $C$ agents). Once in steady states, $D$ agents are passive local maxima, and have reached the maximum payoff allowed by its neighborhood. $C$ agents, do not have such a mechanism and only those agents with sufficiently large payoff may also increase its payoff (by Proposition 3). The overall effect is that in
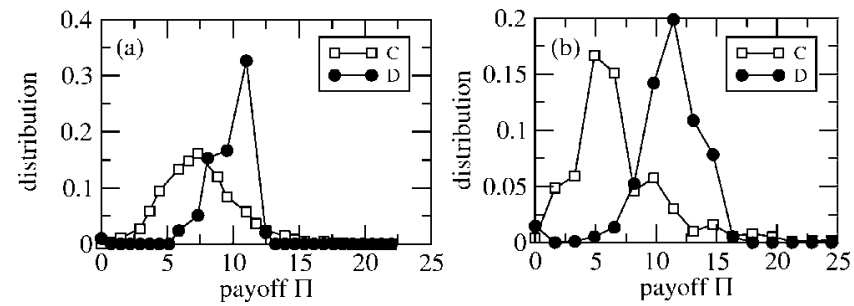

FIG. 8. Distribution of payoffs for cooperators (empty squares) and defectors (filled circles) in a steady state (normalized by the number of cooperators and defectors, respectively). Parameter values: $p=0.08, K=8$, for (a) $b=1.25$ and (b) $b=1.75$.

general we observe that $D$ agents are wealthier than $C$ agents. In other words, our numerical findings points that with network adaptability both populations of agents may be better off in a steady state: $C$ agents may become more numerous, but on average are poorer than defectors.

More detailed numerical simulations show that the unevenness in wealth distribution occurs in particular in the region of incentive to defect $b$ where Nowak and May obtained only partial cooperation. As a measure of uneven wealth distribution, consider the computation of the average payoff for $C$ and $D$ agents at steady state, labeled $\Pi_{C}$ and $\Pi_{D}$, respectively. Figure 9 shows how the average over different realization of $\Pi_{D}-\Pi_{C}$ behaves as a function of $b$. In general we find that on average defectors have a better profit than cooperators. For small $p=0.01,0.1$ (slow convergence to a steady state) the uneven wealth distribution is observed for the whole range of $b$. For $p=1$ (very fast convergence to a steady state) the result holds for most of the range of $b$. There seems to be a relation between the speed a steady state is reached and the possibility of $D$ agents to improve on average their payoff past the $C$ agents' payoff.

\section{Leaders and topology of cooperators clusters}

The uneven wealth distribution found in the previous section may seem a priori a contradiction from the point of view of standard game theory. However, we find that the sustainability of our networks depends crucially on the payoff relation among the $C$ and $D$ agents with largest payoff. In this section we present more detailed numerical results, which reveal some other leaders' properties.

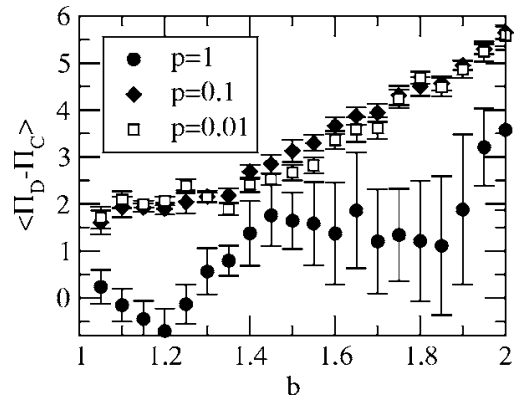

FIG. 9. Payoff difference between average $D$ agent and average $C$ agent as a function of $b$. Parameter values: $K=8$. Data have been averaged over 50 realizations. 


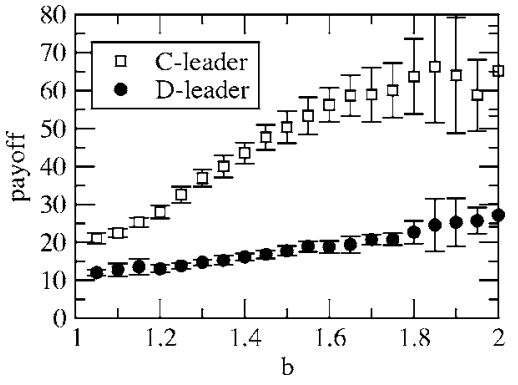

FIG. 10. Payoff of $C$ and $D$ leaders as a function of $b$. Parameter values: $p=1.0, K=8$, averaged over 30 realizations.

An illustration of the maximum payoff $C$ agents and defectors may reach in a steady state is shown in Fig. 10. We have performed a numerical study varying the incentive $b$, with fixed $p=1.0$ and $K=8$, averaging over 30 realizations. We find a steady growth with $b$ for the maximum payoff of both $C$ and $D$ leaders. Also, due to the imposed conservation of the total number of links in our model, the maximum payoff acquired by $C$ leaders depends on the average degree $K$ of the network: for large values of $K, C$ leaders may acquire larger payoffs.

In a steady state, the structure of the network changes as a function of $b$. On the one hand, we have computed the number of $C$ local maxima which are all candidates of being $C$ leaders. Figure 11(a) shows the results. For low $b$ there is a large fraction of competing local maxima in the steady state, while for large incentive $b$ their number is reduced drastically reaching approximately $20 \mathrm{C}$ agents or roughly $0.2 \%$. the whole network. On the other hand, we have measured the relative size of the cluster of the agents connected to the $C$ leader as a function of $b$. Figure 11(b) shows that for high $b$ approximately half of the population is associated with that of the leaders cluster. These results indicates that for larger $b$, the structure of the network depends very strongly on the robustness of only a few local maxima with a very large payoff, and the $C$ leader dominates at least half of the $C$ population.

A typical network structure may be obtained by displaying only the links corresponding to the neighbors of $C$ agents from which they imitate. Figure 12 offers such view. The local maxima are observed to lead large clusters of $C$ agents. It is clear that an exogenous perturbation flipping the strategy
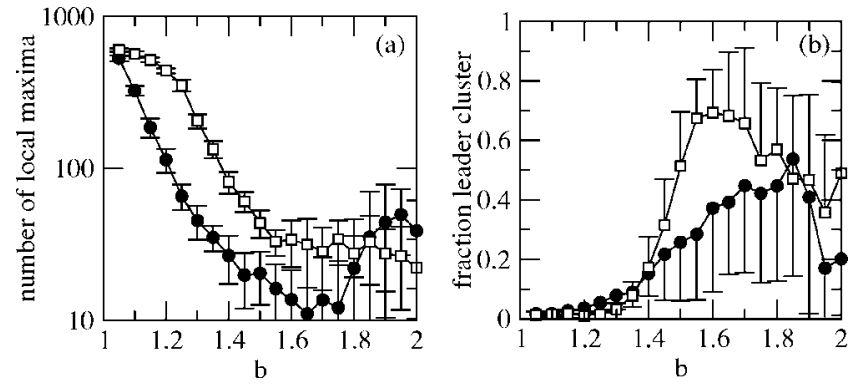

FIG. 11. Leaders' characteristics for $K=8$ and $p=0.01$ (empty squares), and 1.0 (filled circles). (a) Number of local maxima in a steady state. (b) Fraction of agents which belong to the $C$ leader cluster. Data have been averaged over 30 realizations.

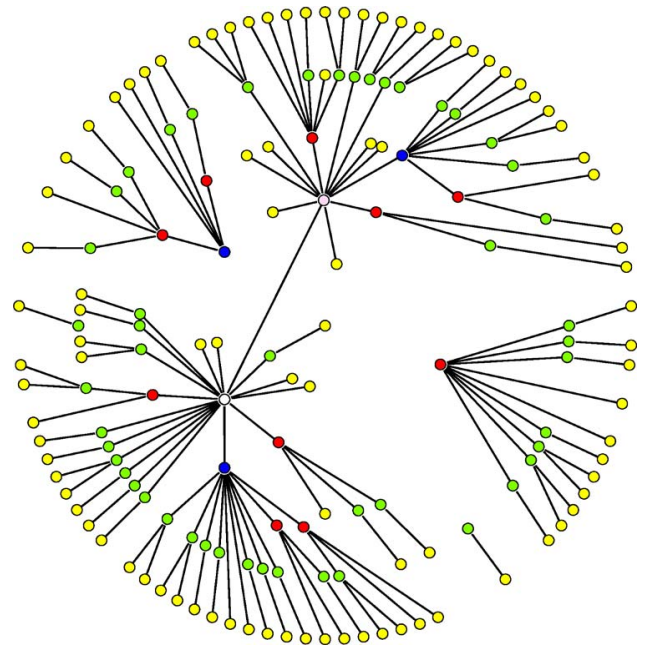

FIG. 12. (Color online) Partial network displaying only $C$ agents and the links corresponding to the evolution of the imitation map $l(i)$ for each agent. The most connected agent corresponds to a $C$ leader. Agents without a link have been withdrawn. Parameter values: $N=900, b=1.45, p=0.03, K=8$. Adapted from Ref. [22].

of any of the local maxima will result in a large dropout of cooperation in very few time steps. This will affect of course other chains due to the links not shown in Fig. 12. Thus the vulnerability of the network to stochastic perturbations is dramatically increased for large $b$.

Finally, in the discussion on chains robustness (Sec. III E), we argued that in order for uphill perturbations not to occur, the "payoff gaps" between neighboring agents had to be sufficiently big (see Table I). A closer look at the resulting chains on any particular steady states reveal that in general "gaps" are very frequent and large. In Table II we reproduce the payoffs for several chains on a particular steady state. The results show that indeed at steady states gaps for agents with high payoff develop. In the next section we will relate this result with the occurrence of avalanches and large cascades.

\section{E. Leaders and global cascades}

We now turn to a detailed discussion of the role of leaders in the global cascades described above. As before, we denote the $C$ agent with maximal payoff by $\alpha$, and the $D$ agent with maximum payoff by $\beta$.

Proposition 3(a) showed how the payoff of a $C$ leader is a nondecreasing function of time, as long as it is larger than the payoff of $\beta$. Figure 13 illustrates a numerical simulation

TABLE II. Examples of chains of cooperators for $b=1.55$.

\begin{tabular}{ccccc}
\hline \hline$K_{i}^{\mathrm{CC}}$ & $K_{l(i)}^{\mathrm{CC}}$ & $K_{l^{2}(i)}^{\mathrm{CC}}$ & $K_{l^{3}(i)}^{\mathrm{CC}}$ & $K_{l^{4}(i)}^{\mathrm{CC}}$ \\
\hline 7 & 12 & 15 & 21 & 37 \\
5 & 13 & 16 & 24 & 38 \\
5 & 13 & 21 & 26 & 38 \\
9 & 14 & 17 & 32 & 38 \\
\hline \hline
\end{tabular}




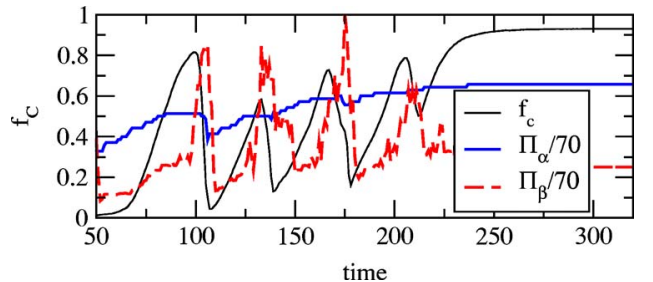

FIG. 13. (Color online) Time evolution of the fraction of cooperators $f_{\mathrm{C}}$ and the rescaled maximum payoff of $C\left(\Pi_{\alpha}\right)$ and $D$ agents $\left(\Pi_{\beta}\right)$. Parameter values: $b=1.75, p=0.08, K=8$.

where the (rescaled) payoff of the $C$ and $D$ leaders are shown along with $f_{\mathrm{C}}$ as a function of time, where several large global cascades developed before settling into a cooperative steady state at $T \approx 300$. The simulation illustrates the leaders payoff increase on each period where $\Pi_{\alpha}>\Pi_{\beta}+b$. Moreover, we find that whenever $\Pi_{\alpha} \approx \Pi_{\beta}$, the maximum (minimum) of $f_{\mathrm{C}}$ is approximately reached if $\Pi_{\beta}$ increases (decreases). The destructive perturbations dominate when $\Pi_{\alpha}$ $<\Pi_{\beta}$, and may even affect the maximum $C$ agent; see, for example, at $T=106$, where a drop of $\Pi_{\alpha}$ is observed. It remains to be explained what is the mechanism by which a $D$ leader may increase its payoff past a $C$ leader.

To answer this question we performed a close inspection to the evolution of a $D$ leader. Our results show that it is the occurrence of uphill perturbations on a chain of cooperators connected to the $C$ leader, which allows in very few time steps for a $D$ leader to increase its payoff past the $C$ leader. As discussed in Sec. III E, uphill perturbations are inhibited if the payoff "gap" between $C$ agents in a chain is sufficiently large [see Eq. (13)]. This boundary where uphill perturbations are inhibited increases linearly with $b$, thus for large $b$ these perturbations will appear more frequently. In accordance, we have found from numerical experiments that for $b>1.45$ and $p>0.01$ large global cascades could easily be observed, and it became easier for larger $b$.

Consider a chain of cooperators whose local maximum is the $C$ leader and the whole chain allows the development of uphill perturbations. Alternatively the discussion is also valid for a chain associated to a local maximum with large payoff. As a $D$ agent perturbs this chain at one point, uphill perturbations will create a "wave" of new $D$ agents moving along the chain with larger and larger payoff (see for a schematic simulation Fig. 3). At some point, the payoff of these newly created $D$ agents will become so large that they will effectively become an uphill "wave" of new $D$ leaders. This phenomenon is visually observed in Fig. 13 , where at $T \approx 100$ the slope of the $D$ leader's payoff changes abruptly. Also note that the maximum payoff attained by a $D$ leader at any oscillation is approximately $b \Pi_{\alpha}$, which occurs when the $C$ leader $\alpha$ is perturbed by an uphill perturbation becoming a $D$ leader. Whenever a $C$ leader is perturbed, then a new $C$ leader with less payoff is selected, as, for example, at $T$ $\approx 105$ in Fig. 13 .

Once an uphill perturbation affects a $C$ leader (or a local maximum with a similar payoff), downhill perturbations will start. This will generate a large global cascade replicating $D$ strategy on all the remaining chains associated to this local maximum. Unlike the uphill perturbations which occurs at a rate proportional to the network adaptation $p$, this process occurs deterministically on every time step until the whole cluster of $C$ agents disappears. Also, the evolution of the $D$ leader follows closely the rapid decrease of the fraction of cooperation. On each time step of the downhill replication of $D$ strategy, there is a $D$ leader $\beta(t)$ which will have a lower payoff than the $D$ leader at the previous time step $\beta(t-1)$. This occurs because at time $t$, the previous $D$ leader replicated its strategy to all its $C$ neighbors, reducing completely its payoff. Thus the payoff of $D$ leaders decreases steadily $\Pi_{\beta}(t)<\Pi_{\beta}(t-1)$ until a new $D$ leader from another chain is selected.

The subsequent time steps after the leader's cluster replicates $D$ strategy depends on the network structure left by the global cascade. If another $C$ leader exists with a payoff such that $\Pi_{\alpha}>\Pi_{\beta}+b$, then cooperation may be recovered and the time evolution of $f_{\mathrm{C}}$ shows a minimum. Otherwise, the $C$ leader may be affected by more destructive perturbations driving it towards the payoff lower boundary [Proposition 3(b)] where $C$ chains may survive. This may drive the whole $C$ population to extinction, reaching the full-defective state.

If the fraction of cooperation reaches a minimum, cooperation may build up again. However, we find that the number of oscillations is finite, and the system either reaches a steady state of the all- $D$ network. We have already described two mechanisms by which global cascades will be hindered and a steady state may be reached:

(a) We have already seen in the discussion after Proposition 3(a) that the existence of "wealthy" $C$ agents such that $\Pi_{i}>\Pi_{\beta}+b$, will absorb a number of constructive perturbations, which effectively decrease the number of links between unsatisfied $D$ agents.

(b) By Proposition 4 the payoff gaps for all wealthy agents is nondecreasing, making it possible to "protect" the $C$ leader from uphill perturbations. Therefore the number of "dangerous" chains which favor uphill perturbations right up to the Cleader decreases.

Clearly from the above discussion, both effects disappear whenever $\Pi_{\alpha} \approx \Pi_{\beta}$, and the "protection mechanism" to $C$ leaders from uphill perturbations ceases. If a dangerous chain is perturbed then large global cascade is ignited, which in very few time steps affects a large fraction of the whole network. On the other hand, if protection of the $C$ leader was guaranteed, then there would be no dangerous chains to perturb. In this case, the maximum attainable payoff the uphill perturbations may reach without affecting the leader is also $\Pi_{\beta} \approx \Pi_{\alpha}$. An example of this is observed in Fig. 13 at $T$ $=206$, where the perturbation did not affect the $C$ leader at all, and the size of the cascade was small. By mechanism (a) above, the network reached a steady state by a steady reduction on the available unsatisfied $D$ agents' links.

We have performed several other computer simulations to visualize different dynamic evolution of the network for various values of $p$ and $b$. For small values of the adaptability parameter $p$, as shown in Fig. 14(a), the typical time for the network to reach a steady state is very large, and the leader $\alpha$ increase its payoff very slowly. The fraction of cooperators increases on average as the leader increases its number of links, and only small cascades are shown. In this case, the network has many local maxima, and the cascades do not affect a large portion of the network. 

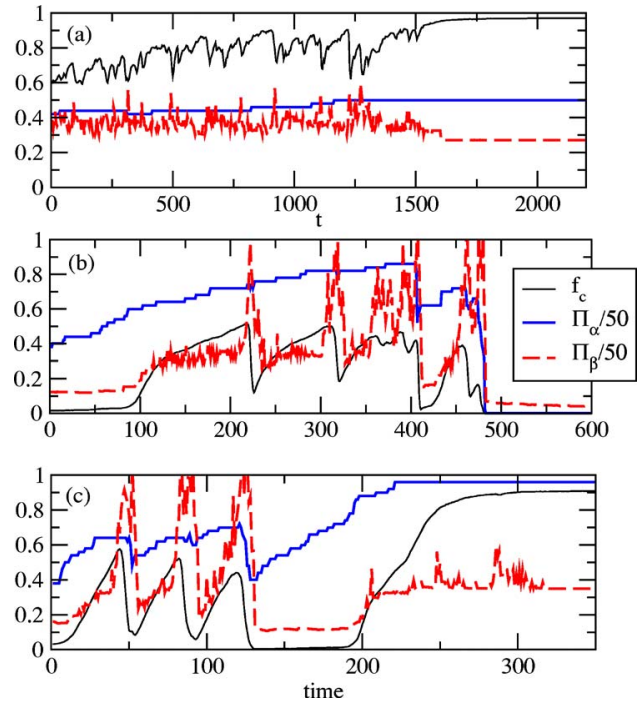

FIG. 14. (Color online) Time evolution of $f_{\mathrm{C}}$ and the rescaled payoff of the $C$ agent $\left(\Pi_{\alpha}\right)$ and the $D$ agent with maximum payoff $\left(\Pi_{\beta}\right)$. Parameter values: $K=8$, (a) $p=0.005, b=1.35$; (b) $p=0.01$, $b=1.75$; and (c) $p=0.05, b=1.75$.

For a higher value of $b$, the structure of the network has wealthier local maxima but also fewer, so large cascades are possible. Figure 14(b) shows that the fraction of cooperators grows steadily, until an uphill perturbation wipes off the cluster, affecting most of the network. This particular run also illustrates how even after having a large $\Pi_{\alpha}$ the asymptotic solution is the all- $D$ network. The key factor to sustain cooperation is to have several local maxima, with large payoff, which are able to survive cascades.

A very interesting situation occurs in Fig. 14(c) at $t$ $\approx 150$, where the network is composed of mostly unsatisfied $D$ agents together with a very wealthy leader $\alpha$ (which at least has sufficient neighbors to keep up its payoff). Note how the leader is able to increase its number of links by the intense (unsuccessful) "searching" done by $D$ agents, and by $t \approx 200$ the fraction of cooperators also increases. This recovery is a clear example of the importance of a wealthy leader, which enables a full cooperative final outcome. Also note that small size uphill cascades for $t>200$ did not reach the $C$ leader and wealthy agents just absorbed all the unsatisfied $D$ agent links.

\section{F. Noise and errors}

In typical evolutionary game theory studies, one is interested to contrast the main results of the original game to those from another perturbed game, which takes into account random variations of the assumed rationality, i.e., errors are possible.

To this end we have modified our model to account for two different types of noise. The first type assumes the imitation process is not perfect and there is a probability $p_{\text {err }}$ that the opposite strategy is imitated. The second type of noise consists of taking at each time step a fraction $p_{\text {rand }}$ of agents and randomize their strategies. Thus on average there is a

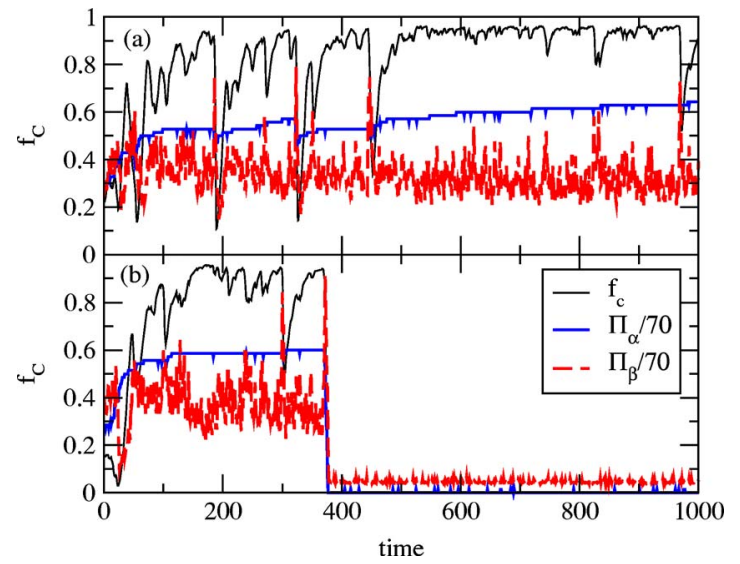

FIG. 15. (Color online) Time evolution of $f_{\mathrm{C}}$, and rescaled maximum payoff of $C$ and $D$ agents when the imitation has a probability $p_{\text {err }}=0.1 \%$ of making an error copying the strategy at each time step: (a) $b=1.45$, (b) $b=1.55$. Parameter values: $K=8, p$ $=0.08, \delta=0.1$.

probability $p_{\text {rand }}$ that the agent switches spontaneously its strategy.

In both cases we find that for small $p_{\text {err }}$ or $p_{\text {rand }}$ there is a region of the incentive to defect $b$ where cooperation is sustained, but now there is a critical value $b^{*}$ where defection sets in. The nature of the transition is that these perturbations affect those agents with highest payoff, which are known to sustain cooperation. Also we have seen that after a large cascade, the cooperative state may be recovered if there is a $C$ leader with sufficiently large payoff. The speed at which the cooperative state forms depends on the network adaptation rate $p$ and the noise intensity, which introduces further randomness. Therefore there is always a sufficiently strong noise intensity which perturb most $C$ local maxima before they ever regenerate, driving the system to the all- $D$ network.

We display in Fig. 15 two time series of $f_{\mathrm{C}}$ and the payoff of the maximal $C$ and $D$ agents, showing the two possible asymptotic states. Both panels show how the $C$ leader is affected by the noise perturbations, and new $C$ local maxima take its place. In Fig. 16 we display how the fraction of cooperation changes as $b$ is increased for fixed values of the

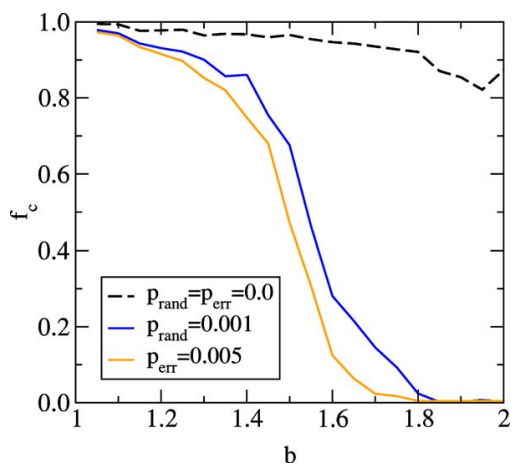

FIG. 16. (Color online) Fraction of cooperation vs $b$ for different types of noise. $p_{\text {err }}$ corresponds to the probability that imitation reads the opposite value, while $p_{\text {rand }}$ is the probability that an agent is selected and its strategy randomized. Data have been averaged over 100 realizations $(p=0.08, K=8, T=500)$. 
$p_{\text {err }}$ and $p_{\text {rand }}$. The value of the sharp transition depends on that of the chosen noise intensities.

\section{DISCUSSION}

Social and economical interactions are frequently based on the ability of the agents to choose and refuse partners depending on their own interests. In this paper we have proposed, assuming the bounded rationality of agents, a framework that naturally incorporates such possibility. We have presented numerical and theoretical results of a Prisoner's Dilemma game played in an adaptive network, where the agents have the possibility to change their local neighborhood depending on their level of satisfaction and the strategy played.

In other recent studies of network evolution the adaptation does not depend on the outcome of a game, but instead links are created or destroyed according to a balance between benefit and cost these represent $[10,27,33,34]$. The paper of Ref. [10] studies network formation where the costs incurred to form a new link is taken only by one of the agents. The agent receiving a link does not incur in a cost and just shares the benefit. If the link benefits both agents, then it is established, otherwise it is discarded. In our case, we have followed a different approach. Our agents cannot assess a priori if the receiving link will be entirely beneficial or not, but as it is costless, it is willing to accept it. Also as only $D$ agents are responsible for the initiative of neighborhood adaptation, they are mostly interested in finding a $C$ agent to exploit. Thus in order to avoid a generation of (costless) links, we specifically study an evolution where the number of links is conserved.

Another related work to ours is Ref. [35], where the network is not assumed a priori, but is determined endogenously from expected payoffs agents have across them. If this payoff exceeds a given threshold, then the agent makes an offer to play with that neighbor; next, it revises all offers it receives and refuses those which are below his threshold. Therefore the game is carried out on a network which is formed in a preplay stage, depending on the outcome of the previous game. Their main result also includes that cooperation is highly enhanced, although a steady state is never reached, for exploiter $D$ agents migrate continuously among $C$ agents. Our approach assumes agents' computation capabilities to be much more restrictive, and $C$ agents cannot explicitly refuse to play with $D$ agents. In our model steady states display $D$ agents exploiting $C$ agents. This results from two assumptions: (i) satisfied $D$ agents do not change their neighbors, and (ii) the desire a $C$ agent has to dismiss an interaction with a $D$ neighbor exactly balances the effort of the $D$ neighbor to maintain the interaction.

Our approach focuses on a specific coupling between the network adaptation mechanism and the agents' strategy. Cooperators play a conservative role, while unsatisfied $D$ agents search for new $C$ agents in order to improve their payoff. The searching stops whenever all $D$ agents become satisfied, reaching a steady state configuration. In some sense, the network adaptability is analogous to a mutation process (as in the traditional evolutionary game theory) to the network, which succeeds in finding an "optimal" compromise to the roles taken by the agents. This solution makes (i) $D$ agents in steady state become satisfied, with a higher average payoff (with respect to $C$ agents), and (ii) $C$ agents are in general unsatisfied (but conformists), with a lower average payoff. Very remarkably, considering the average payoff of agents, the social dilemma holds, because on average defectors are wealthier than cooperators.

In a more detailed description, the searching capabilities of defectors has an interesting effect towards the evolution of network "leaders." The $C$ leader is selected among those $C$ local maxima with largest payoff. These special agents are responsible of sustaining cooperation, for whenever their payoff is larger than the $D$ leader, its payoff increases by accommodating new $C$ neighbors.

Our numerical findings show that as a result of the network adaptation, the network topology becomes more hierarchical as $b$ increases (the wealth of $C$ local maxima increases, while the number of them in steady states decreases). In this case the emergence of wealthy leaders makes the sustainability of cooperation rely heavily on them, especially under some destructive endogenous perturbations produced by searching $D$ agents. These special perturbations may occur for given network configurations, through "dangerous chains," and may affect a $C$ leader. When this happens, global cooperation is temporally abandoned and a large cascade of $D$ strategy replication is started. However, the payoff of $D$ agents soon drops until once again cooperation slowly recovers when the $C$ leader is the wealthiest agent in the whole network. If the latter does not happen, then by more destructive perturbations there is the probability that the system reaches the all- $D$ network. We also remarked that there exists a mechanism by which the dangerous chains in the network decrease in number, providing the possibility that the leader may be well protected and inhibiting large cascades.

By adding the "degree of freedom" of network adaptation, we were able to explore how well established results in cooperation theory with fixed neighborhoods might be affected. Cooperation among a set of selfish agents is possible in an adaptive local neighborhood, even when the time scale of network adaptation is slow compared with the strategy update.

Finally we mention the work of Ref. [11] which shows the relevance of context preservation, i.e., dynamics where the neighborhood remained unaltered get better average payoff than those dynamics that do not preserve the neighborhoods. The framework we have presented in this paper allows us to study a different dynamics with variable adaptation. Our results show that cooperation can be sustained even in situations where the neighborhoods are not fully preserved, with the fraction of cooperation being similar to the preserved one. However, our model differs from the situations studied by Ref. [11] in which the adaptation of the network is independent of the process of decision making, i.e., there is no feedback from the payoff to the network evolution. In our framework, the feedback between payoff and network adaptation is clear: the adaptation of the network only takes place locally for the unsatisfied agents. This difference is fundamental to explain the results we have obtained. 
Many social interactions can be modeled in the framework of networks with adaptive interactions: new interactions are created or suppressed depending on the benefits obtained by the agents taking part on the interaction. Collaboration networks in science is an example of a social system that could be modeled with this approach. While we have shown that cooperation can be sustained in an adaptive network, a more realistic version of the model aiming to capture also the stylized features of scientific collaboration networks should include some sort of preferential search for new partners. Work along these lines is in progress.

\section{ACKNOWLEDGMENTS}

We acknowledge discussions with Maxi San Miguel, and financial support from the CONICET and ANPCYT (Argentina), CONOCE2, and FIS2004-05073-C04-03 (Spain).
[1] M. G. Zimmermann, V. M. Eguíluz, and M. San Miguel, Phys. Rev. E 69, 065102(R) (2004).

[2] J. Weibull, Evolutionary Game Theory (MIT University Press, Cambridge, MA, 1996).

[3] R. Axelrod and W. D. Hamilton, Science 211, 1390 (1981).

[4] A. Kirman, Revue Européenne des Sciences Sociales 113, 189 (1999).

[5] A. Bannerjee, Quart. J. Econom. 108, 797 (1992).

[6] R. Cont and J. P. Bouchaud, Macroecon. Dyn. 4, 170 (2000).

[7] V. M. Eguĺluz and M. G. Zimmermann, Phys. Rev. Lett. 85, 5659 (2000).

[8] A. Kirman, Quart. J. Econom. 108, 137 (1993).

[9] C. A. Ferrer, A. B. Ania, and F. Vega-Redondo, in The Theory of Markets, edited by J.-J. Herings, A. Talman, and G. van der Laan (North-Holland, Amsterdam, 1999).

[10] S. Goyal and S. Joshi, Games Econ. Behav. 43, 57 (2003).

[11] M. Cohen, R. Riolo, and R. Axelrod, Santa Fe Institute, Working Paper 99-01-002.

[12] B. A. Huberman and N. S. Glance, Proc. Natl. Acad. Sci. U.S.A. 90, 7716 (1993).

[13] K. Lindgren, in The Economy as an Evolving Complex System II, SFI Studies in the Sciences of Complexity Vol. XXVII, edited by W. B. Arthur, S. N. Durlauf, D. A. Lane (AddisonWesley, Reading, MA, 1997), pp. 337-369.

[14] K. Lindgren and M. G. Nordahl, Physica D 75, 292 (1994).

[15] M. A. Nowak and R. M. May, Nature (London) 359, 826 (1992).

[16] M. A. Nowak and R. M. May, Int. J. Bifurcation Chaos Appl. Sci. Eng. 3, 35 (1993).

[17] O. Kirchkamp, J. Econ. Behav. Organ. 43, 239 (2000).

[18] M. E. J. Newman, Proc. Natl. Acad. Sci. U.S.A. 98, 404 (2001).

[19] H. Jeong, Z. Neda, and A.-L. Barabási, Europhys. Lett. 61, 567 (2003)

[20] A.-L. Barabási, H. Jeong, Z. Neda, T. V. E. Ravasz, and A. Schubert, Physica A 311, 590 (2003).

[21] M. G. Zimmermann, V. M. Eguíluz, and M. San Miguel, in Economics with Heterogeneous Interacting Agents, Lecture
Notes in Economics and Mathematical Series No. 503, edited by A. Kirman and J.-B. Zimmermann (Springer, Berlin, 2001), pp. 73-86.

[22] V. M. Eguíluz, M. G. Zimmermann, C. J. Cela-Conde, and M. San Miguel, Am. J. Sociol. 110, 977 (2005).

[23] Numerical simulations with asynchronous update have been performed and compared to the results of Fig. 7, with no noticeable difference.

[24] H. P. Young, Brookings Institution, CSED Working Paper No. 2.

[25] L. E. Blume, Games Econ. Behav. 5, 387 (1993).

[26] O. Kirchkamp and K. H. Schlag (unpublished).

[27] A. Watts, Games Econ. Behav. 34, 331 (2001).

[28] D. Hirshleifer and E. Rasmussen, J. Econ. Behav. Organ. 12, 87 (1989).

[29] Note that in the following, we assume that the elements of $\mathcal{V}(j)$ do not have any other direct link to the chain in question $\xi$. However, the perturbation $j$ may affect other chains as well, which in turn may affect $\xi$. To simplify matters we do not discuss this effect, which will be negligible when we work with a large number of agents $N$, and when we are sufficiently "close" to a steady state.

[30] In Refs. [15,31] regular lattices were used, but not a strict Prisoner's Dilemma game because they considered the PD parameter $\delta=0$. In this case the one-shot game does not have a unique Nash equilibrium. Despite this multiplicity of equilibria, the fraction of cooperators is analogous to the results obtained in Fig. 7. See similar results in Refs. $[1,17]$.

[31] G. Szabó and C. Töke, Phys. Rev. E 58, 69 (1998).

[32] K. Lindgren, in Artificial Life II, SFI Studies in the Sciences of Complexity Vol. X, edited by C. G. Langton, C.Taylor, J. D. Farmer, and S. Rasmussen (Addison-Wesley, Longman, 1999), pp. 295-312.

[33] M. O. Jackson and A. Watts, J. Econ. Theory 106, 265 (2002), vanderbilt University, Mimeo.

[34] V. Bala and S. Goyal, Econometrica 68, 1181 (2000).

[35] D. Ashlock, M. D. Smucker, E. A. Stanley, and L. Tesfatsion, BioSystems 37, 99 (1996). 